\title{
Simulation of surface-enhanced ordering in smectic films
}

\author{
Nasser Mohieddin Abukhdeir ${ }^{1, a}$, Alejandro D Rey ${ }^{1, b}$ \\ ${ }^{1}$ McGill University - Dept. of Chemical Engineering \\ 3610 University St. \\ Montreal, Quebec H3H 2B2, Canada \\ anasser.abukhdeir@mcgill.ca, balejandro.rey@mcgill.ca
}

Keywords: liquid crystal mesophase surface ordering smectic-A lamellar layer

\begin{abstract}
A Landau-de Gennes type model for the direct isotropic/smectic A phase transition is used to study surface-enhanced smectic ordering in the stable smectic temperature regime. A unified surface-free energy functional is proposed which can be utilized for homeotropic and planar surface anchoring. The time-dependent complex Landau-Ginzburg evolution equations and boundary conditions are derived for thin-film geometry. Simulation results are presented for the two types of anchoring and compared to observations from experiments and previous simulations. Simple visualization software for smectic layering was developed and is also presented that is compatible with discretized numerical solutions of the model.
\end{abstract}

\section{Introduction}

Surface enhanced ordering is a pervasive phenomenon in the study and application of liquid crystal systems. The vast majority of applications involving liquid crystals either directly or indirectly rely on interfacial properties. These surfaces effect the molecular ordering in the neighborhood of the surface which also determines the texture formation in the bulk. An understanding of surface effects includes a broad range of applications including lubrication [1], display technology, flexible polymer crystallization [2] et al.

Experimentally observed surface induced mesophase transitions have been studied mainly for the isotropic/nematic transition series [3-5]. The nematic/smectic A and isotropic/smectic surface induced transitions have been studied less [1,6-10]. The isotropic/smectic A transition is of great practical interest because of the its appearance in sheared flexible polymer melts. Thus the further understanding of the effect of free surfaces on the smectic A phase moves one step closer to other fundamental problems such as the core structure of smectic defects and nucleation/growth of the smectic phase.

Liquid crystals encompass a diverse set of molecules that exhibit some degree of anisotropic molecular order in the appropriate physical setting. Thermotropic liquid crystals exhibit mesophase ordering depending mainly on temperature while lyotropic liquid crystals strongly depend on concentration (and to a lesser extent temperature).

Of the many types of liquid crystalline order two are focused on in this work. The simplest type is nematic order where the molecules exhibit orientational order. Typically this order is characterized by a symmetric and traceless tensor $\mathbf{Q}=\mathbf{S}(\mathbf{n n}-\mathbf{I} / 3)$ [11]. This tensor is composed of a scalar $\mathrm{S}$, the scalar nematic order parameter, and a unit vector $\mathbf{n}$, the nematic director. The nematic director is parallel to the average orientational axis and is a measure of long range order. The scalar nematic order parameter characterizes the extent to which the molecules conform to the director and is a measure of short range order. Thus models using the Q-tensor such as the Landaude Gennes model are mesoscopic models.

The next more complex mesophase exhibits one-dimensional positional order in addition to orientational order. It is called the smectic A phase and can be visualized as planes of positional isotropic liquid stacked upon each other. Characterization of this density modulation uses the complex order parameter in addition to the $\mathbf{Q}$ tensor [11]. The complex order parameter, 
$=\psi \exp (\varphi \mathrm{i})$, is composed of short and long range terms. The scalar smectic order parameter $\psi$ is the magnitude of the density wave and $\mathbf{a}=\nabla \varphi /|\nabla \varphi|$ is the layer normal unit vector, or wave vector.

\section{The Model}

A Landau-de Gennes type model for the isotropic/smectic A phase transition is used that was presented by Mukherjee, Pleiner, and Brand [12,13]. We extend the model by adding a nematic elastic term, $1_{1}$, to model smectic defects [14-16] in addition to nucleation and growth. An important aspect of the model is the inclusion of a second order derivative term of the complex order parameter. This term directly penalizes curvature of the layers. In addition to this, nematic elastic terms are necessary to account for smectic disclinations and interfaces during nucleation and growth [17-19]. The effect of including the $1_{1}, 1_{2}$ and $l_{3}$ is an interesting problem that is currently being studied.

A key motivation for the use of weak surface anchoring (surface free energy) as opposed to strong anchoring (Dirichlet conditions) is that self-selected mesophase ordering at the boundary is physically realistic. This is critically important for further applications of the model including studying surface enhanced pre-ordering in the unstable liquid crystal temperature regime.

Free Energy Equations. The complex order parameter is divided into its real and imaginary parts, $\Psi=\mathrm{A}+\mathrm{Bi}$ and the resulting free energy equation is as follows:

$$
\begin{aligned}
& f_{b}=\frac{1}{2} a(\mathbf{Q}: \mathbf{Q})-\frac{1}{3} b\left(\mathbf{Q}_{i j} \mathbf{Q}_{j k} \mathbf{Q}_{k i}\right)+\frac{1}{4} c(\mathbf{Q}: \mathbf{Q})^{2}+\frac{1}{2} \alpha\left(A^{2}+B^{2}\right)+\frac{1}{4} \beta\left(A^{2}+B^{2}\right)^{2}-\frac{1}{4} \delta(\mathbf{Q}: \mathbf{Q})\left(A^{2}+B^{2}\right) \\
& +\frac{1}{2} l_{1}(\nabla \mathbf{Q}: \nabla \mathbf{Q})+\frac{1}{2} b_{1}\left[(\nabla A)^{2}+(\nabla B)^{2}\right]+\frac{1}{4} b_{2}\left[\left(\nabla^{2} A\right)^{2}+\left(\nabla^{2} B\right)^{2}\right]-\frac{1}{2} e \mathbf{Q}_{i j}\left(\nabla_{i} A \nabla_{j} A+\nabla_{i} B \nabla_{j} B\right) .
\end{aligned}
$$

where terms $1-5$ are the bulk contributions to the free energy with $a=a_{0}\left(T-T_{N I}\right)$ and $\alpha=\alpha_{0}\left(T-T_{A I}\right)$. Term 6 is a quadratic coupling between nematic and smectic ordering. Term 7 is the nematic elastic contribution and terms 8-9 are the smectic elastic contributions. Finally, term 10 is a coupling between long range nematic (director) and smectic ordering (wave vector).

The surface free energy is as follows:

$$
f_{s}=-r_{1} \psi+r_{2} \psi^{2}-r_{3}(\mathbf{H} \cdot \mathbf{Q}): \mathbf{I}=-\frac{1}{2} r_{1}\left(A^{2}+B^{2}\right)^{\frac{1}{2}}+\frac{1}{4} r_{2}\left(A^{2}+B^{2}\right)-r_{3}(\mathbf{H} \cdot \mathbf{Q}): \mathbf{I} \text {. }
$$

where terms 1-2 characterize weak smectic anchoring [1,9] and term 3 weak nematic anchoring [20,21]. In this model $r_{1}, r_{2}>0, r_{3} \geq 0$ is necessary homeotropic anchoring and $r_{1}=r_{2}=0, r_{3}>0$ characterizes planar anchoring. The orientation of the nematic anchoring is determined by the unit tensor $\mathbf{H}$.

In order to simulate thin film geometry, a two-dimensional square computational domain of size $\mathrm{L}^{2}$ is used with periodic conditions at the boundaries not corresponding to a surface. The surfaces contribute two sets of boundary conditions: a constant phase/layer [1,9] and a contribution to the scalar order parameters resulting from the surface free energy minimization.

The phenomenological constants utilized are computed incorporating experimental data from the 12CB liquid crystal [22-24] and using a nonlinear programming formulation to determine a suitable set of coefficients [25].

Time-dependent Formulation. The Landau-Ginzburg time-dependent formulation is utilized in these simulations in order to capture the kinetics of layer formation. Due to the higher order derivative term in the free energy functional the resulting time-dependent equations are reformulated to be compatible with the source/divergence formulation required by the finite element 
package employed. The higher order terms also result in additional boundary conditions [26]. The general form of the time-dependent formulation incorporating the surface free energy is as follows:

$$
\begin{aligned}
& \mu \frac{\partial \eta}{\partial \tau}=-\frac{\delta f_{b}}{\delta \eta}=\frac{\partial f_{b}}{\partial \eta}+\partial_{i}\left(\frac{\partial f_{b}}{\partial\left(\partial_{i} \eta\right)}\right)-\partial_{i} \partial_{j}\left(\frac{\partial f_{b}}{\partial\left(\partial_{i} \partial_{j} \eta\right)}\right) . \\
& \frac{\partial f_{b}}{\partial\left(\partial_{i} \eta\right)}-\partial_{j}\left(\frac{\partial f_{b}}{\partial\left(\partial_{i} \partial_{j} \eta\right)}\right)=\frac{\partial f_{s}}{\partial \eta} . \\
& \frac{\partial f_{b}}{\partial\left(\partial_{i} \partial_{j} \eta\right)}=\frac{\partial f_{s}}{\partial\left(\partial_{i} \eta\right)} .
\end{aligned}
$$

where $\mu$ is a viscosity term, $\tau$ is time, $\eta$ is the order parameter, $f_{b}$ is the bulk free energy, and $f_{s}$ is the surface free energy. Eq. 3a is evaluated over the bulk and Eqs. 3b-c are boundary conditions. An important point in implementing the smectic boundary conditions is that the phase is chose such that $\mathrm{A}=\psi$ and $\mathrm{B}=0$ at the boundaries. Thus Eqs. $3 \mathrm{~b}-\mathrm{c}$ are applied to the $\mathrm{A}$-component and Dirichlet conditions are used for the B-component.

Visualization. A simple visualization package using MATLAB and Comsol scripting was developed to facilitate the display of simulation results. Due to the plethora of data to be displayed for even a simple smectic volume, custom visualization methods greatly facilitate the postprocessing of data. A sample of the output of this software can be seen in Fig. 1. Smectic layers are enclosed layered regions with central dotted lines proportional to the magnitude of the scalar smectic order parameter. Only the director portion of nematic ordering is displayed and the scalar nematic order is not necessary in for use with these results given the lack of smectic disclinations and strong coupling between the director and wave vector. This software will be extended for use as a visualization and analysis tool including: the characterization/display of nematic/smectic defects and of mesophase front growth.

\section{Results and Discussion}

The bulk of the results presented deal with the homeotropic case based upon experimental observations and past theoretical studies [1,6-10]. Fig. 1 shows two different test simulations with a commensurate geometry and initial conditions; the domain length is an integer multiple of the smectic layer spacing. In this case the surface coupling and bulk ordering are compatible with the number of layers (an initial condition) and the system is in a stable state. As a result of the use of periodic boundary conditions, undulation of the layers is restricted to a wavelength less than the length of the computational domain, L. This wavelength is characterized by the smectic correlation length and the domain length [27]:

$$
\lambda_{s}=\sqrt{\frac{b_{2}}{b_{1}}}, \lambda_{u}=\frac{2 \pi}{\sqrt{\frac{\pi}{\lambda_{s} L}}}
$$

where $\lambda_{\mathrm{s}}$ is the smectic correlation length and $\lambda_{\mathrm{u}}$ is the undulation period. The value of $\lambda_{\mathrm{u}}$ at these length scales is on the order of $20 \mu \mathrm{m}$ and the undulation mode does not contribute substantially until larger film thicknesses. A study of this was performed but is out of the scope of this report.

A layer expansion study was performed in order to observe a first-order $n / n+1$ layer transition. A simulation series was performed starting with the stable case shown in Fig. 1a/c where $\mathrm{L} / \mathrm{d}=4=$ the number of layers. At each $\mathrm{L} / \mathrm{d}$ value a stable solution was obtained and used as an initial condition for the following simulation with increased $\mathrm{L} / \mathrm{d}$. Each simulation solution provides time evolution from the initial state to the final stable state. Fig. 2 shows the free 

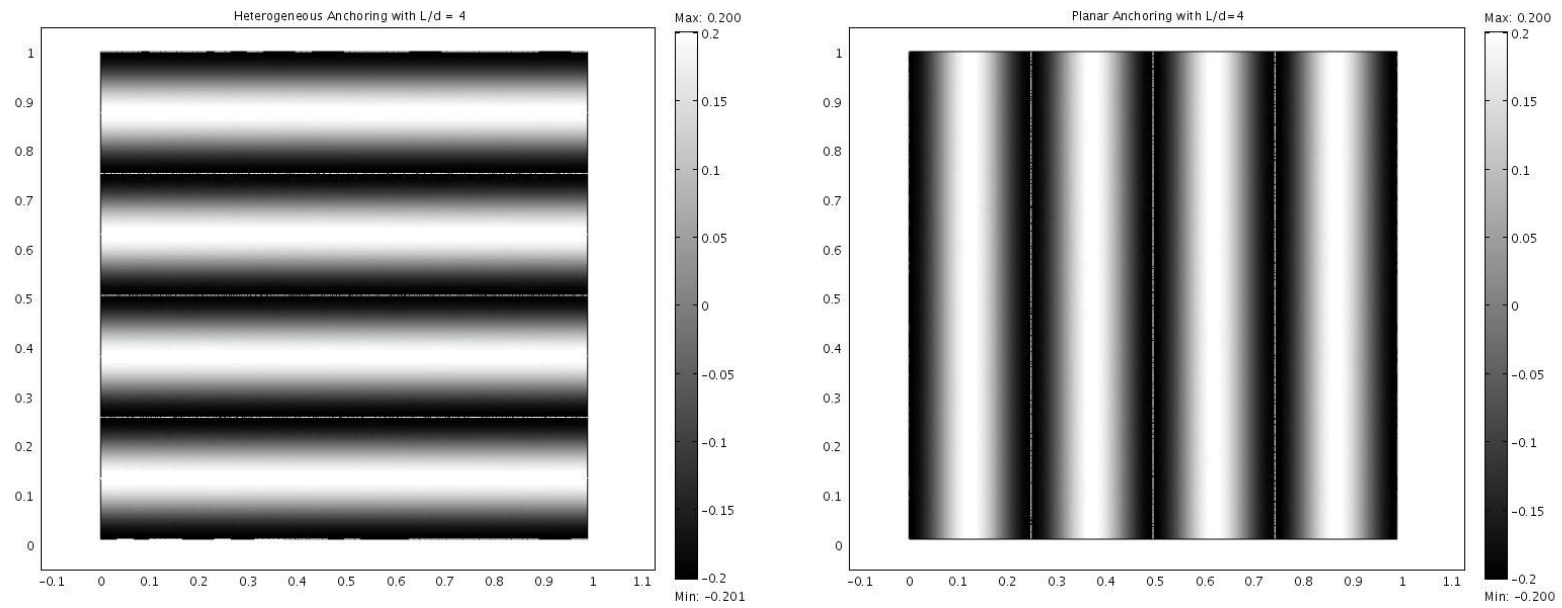

Figure 1: a) $\operatorname{Re}(\Psi)$ and $\mathbf{n}$, homeotropic boundary conditions with $r_{1}=10^{6} \mathrm{~J} / \mathrm{cm}^{2}, r_{2}=2.5 \times 10^{6} \mathrm{~J} / \mathrm{cm}^{2}$, and $r_{3}=0 \mathrm{~J} / \mathrm{cm}^{2} \mathrm{~b}$ ) displayed with the visualization package c) $\operatorname{Re}(\Psi)$ and $\mathbf{n}$, planar boundary conditions with $r_{1}=0 \mathrm{~J} / \mathrm{cm}^{2}, r_{2}=0 \mathrm{~J} / \mathrm{cm}^{2}$, and $r_{3}=10^{4} \mathrm{~J} / \mathrm{cm}^{2} \mathrm{~d}$ ) displayed with the visualization package.

energy results from the stable solutions where, as expected, the behavior deviates from the second order de Gennes layer transition [1]. The mechanism by which the layer transition occurs is in agreement with theoretical predictions as can be seen in Fig. 3. Melting of the smectic order and reformation of a new layer occurs at an $\mathrm{L} / \mathrm{d}$ ratio of 5.3.

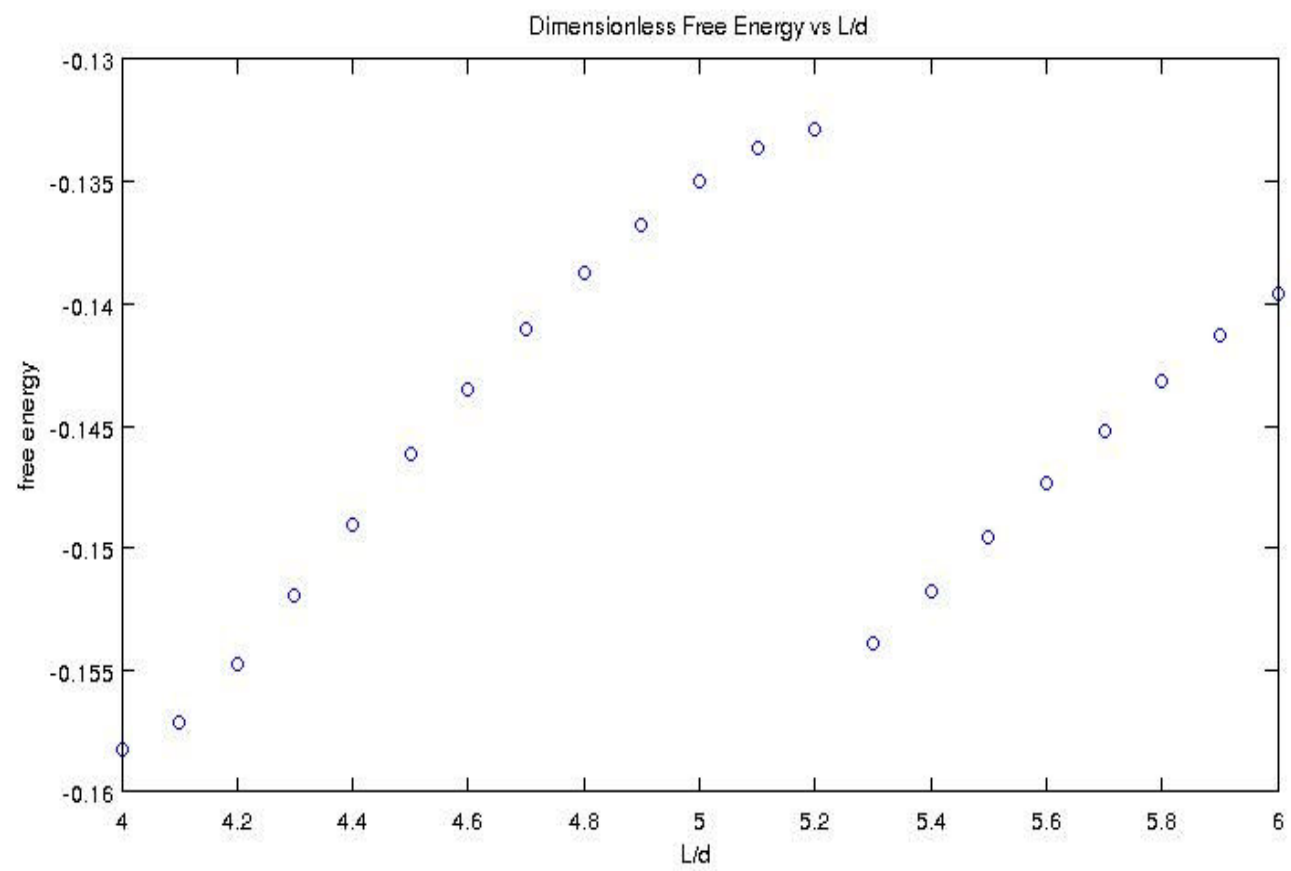

Figure 2: Dimensionless free energy $\mathrm{F} /\left(\alpha_{0} \Delta \mathrm{T}\right)$ versus $\mathrm{L} / \mathrm{d}$, the phenomenological constants used in these simulations are $\mathrm{a}_{0}=1.3333 \times 10^{5} \mathrm{~J} / \mathrm{m}^{3}, \mathrm{~b}=1.2447 \times 10^{7} \mathrm{~J} / \mathrm{m}^{3}, \mathrm{c}=5.5360 \times 10^{6} \mathrm{~J} / \mathrm{m}^{3}, \alpha_{0}=1.9071 \times 10^{6}$ $\mathrm{J} / \mathrm{m}^{3}, \beta=4.1131 \times 10^{8} \mathrm{~J} / \mathrm{m}^{3}, \delta=1.3333 \times 10^{6} \mathrm{~J} / \mathrm{m}^{3}, 1_{1}=10^{-12} \mathrm{~J} / \mathrm{m}, \mathrm{b}_{1}=2 \times 10^{-12} \mathrm{~J} / \mathrm{m}, \mathrm{b}_{2}=4.4394 \times 10^{-30} \mathrm{Jm}$, and $\mathrm{e}=1.8315 \times 10^{-11} \mathrm{~J} / \mathrm{m}$ with $\mathrm{T}_{\mathrm{NI}}=322.85 \mathrm{~K}, \mathrm{~T}_{\mathrm{AI}}=330.5 \mathrm{~K}$, and $\mathrm{T}=330 \mathrm{~K}$.

The melting transition is one of the two mechanisms predicted for an $n / n+1$ layer transition. The second is a mechanism where layers form as a result of dislocation loops. This mechanism was proposed and experimentally observed by Pankratz et al $[28,29]$ and the kinetics of this mechanism are not well understood. Preliminary data shows that the presented model is capable of capturing 
this mechanism as is seen in Fig. 4. Further study is in progress to determine the kinetics of this mechanism and relationship between $\mathrm{L} / \mathrm{d}$ and the $\mathrm{n} / \mathrm{n}+1$ mechanism.

\section{Conclusions}

A proposed surface free energy was used to simulate weak heterogeneous and planer smectic anchoring at a surface. Results were presented showing correct behavior in a commensurate geometry. An $\mathrm{n} / \mathrm{n}+1$ layer transition study was presented showing that the model predicts a first order transition via melting. This result deviates from the ideal second order case

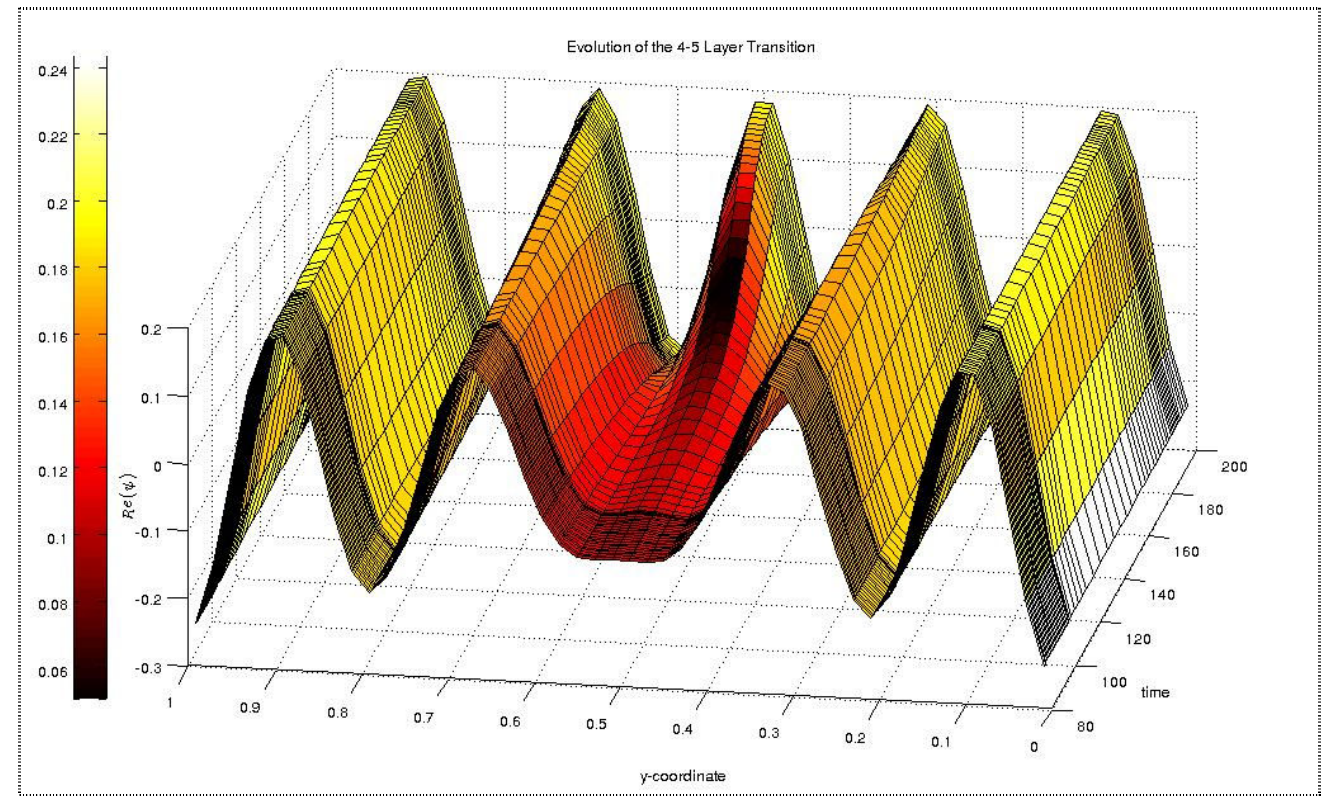

Figure 3: The evolution diagram for 4/5 layer transition where $\mathrm{L} / \mathrm{d}=5.3$ where the shading represents $\psi$, the scalar smectic order parameter.

which is expected for a liquid crystal system exhibiting a first order I/SmA phase transition. Preliminary data shows that this model captures a more complex layer transition mechanism via the formation of dislocation loops. Further study is required to characterize the conditions under which each mechanism is observed and the kinetics of the dislocation loop mechanism.
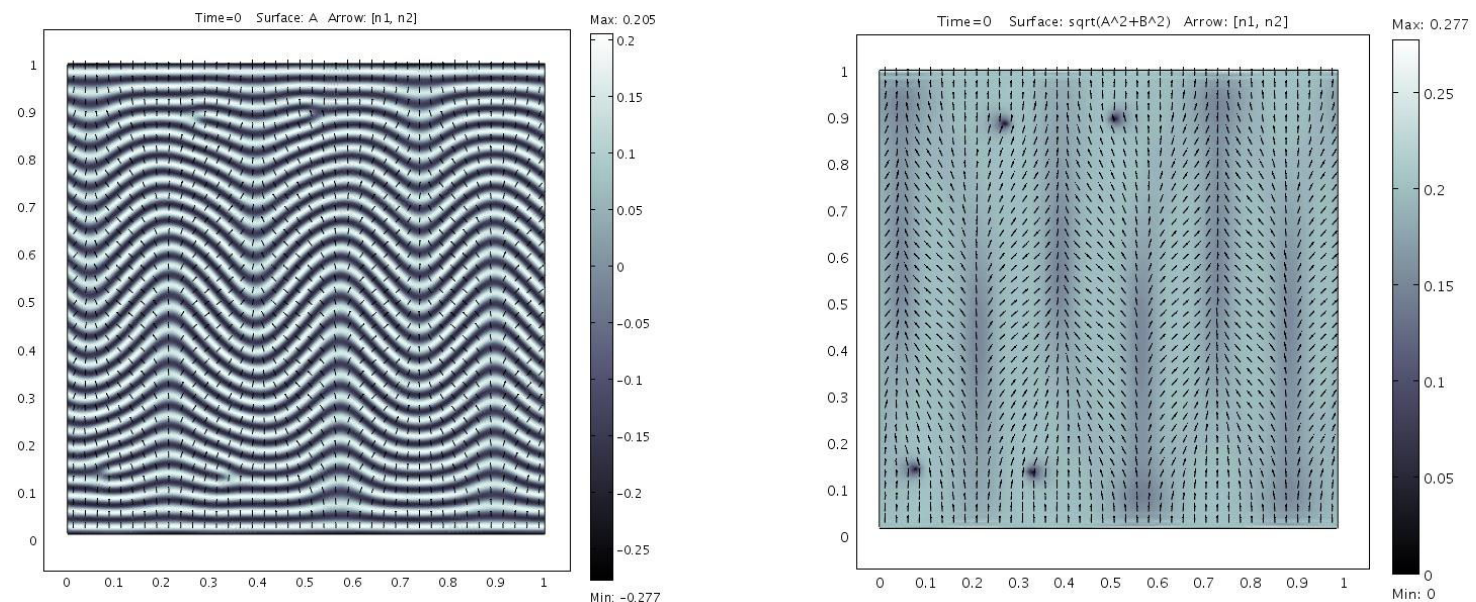

Figure 4: The beginning of a 25/27 layer transition via the dislocation loop mechanism, a) the density wave and $\mathbf{n}$ b) $\psi$ and $\mathbf{n}$. 


\section{Acknowledgements}

The authors would like to thank Benjamin Wincure and Gino De Luca for many useful discussions. Funding was provided a William Gauvin Fellowship and a Natural Sciences and Engineering Research Council of Canada grant.

\section{References}

[1] P. G. De Gennes: Langmuir Vol. 6 (1990), p.1448.

[2] Liangbin Li and W.H. de Jeu: in Interphases and Mesophases in Polymer Crystalliza-

tion II, edited by G. Allegra (Springer, 2005), p. 1-55.

[3] Ping Sheng: Phys. Rev. Lett. Vol. 37 (1976), p.1059.

[4] Ping Sheng: Phys. Rev. A Vol. 26 (1982), p.1610.

[5] Ruibao Tao, Ping Sheng, and Z. F. Lin: Phys. Rev. Lett. Vol. 70 (1993), p.1271.

[6] P. Galatola, M. Zelazna, and I. Lelidis: The European Physical Journal Vol. 2 (1998) p.51.

[7] E. E. Gorodetskii, E. S. Pikina, and V. E. Podnek: Journal of Experimental and Theoretical Vol. 88, (1999) p.35.

[8] J.W. Emsley, G. R. Luckhurst, and P. Pedrielli: Chemical Physics Letters Vol. 320 (2000), p.255.

[9] A. N. Shalaginov and D. E. Sullivan: Phys. Rev. E Vol. 63 (2001), p.031704.

[10] I. Lelidis and P. Galatola: Molecular Crystals \& Liquid Crystals Vol. 413 (2004), p.145.

[11] P.G. de Gennes and J Prost: The Physics of Liquid Crystals, $2^{\text {nd }}$ ed. (Oxford University Press, New York, 1995).

[12] P. K. Mukherjee, H. Pleiner, and H. R. Brand: European Physical Journal E Vol. 4 (2001), p.293.

[13] A. E. Prostakov: Crystallography Reports Vol. 47 (2002), p.856.

[14] J B Fournier: European Journal of Physics Vol. 15 (1994), p.319.

[15] S. Stallinga and G.. Vertogen: Physical Review E: Statistical Physics, Plasmas, Fluids, and Reated Interdisciplinary Topics Vol. 51 (1995), p.536.

[16] P. Biscari, M.C. Calderer, and E.M. Terentjev: Phys Rev E Vol. 75 (2007), p.051707.

[17] B. Wincure and A. D. Rey: The Journal of Chemical Physics Vol. 124 (2006), p.244902.

[18] B. Wincure and A. D. Rey: Continuum Mechanics and Thermodynamics Vol. 19 (2007), p. 37.

[19] B. Wincure and A.D. Rey: Nano Letters Vol. 7 (2007), p.1474.

[20] A. Poniewierski and A. Samborski: Phys. Rev. E Vol. 51 (1995), p.4574.

[21] A. Poniewierski and A. Samborski: Pol. J. Chem. Vol. 75 (2001), p.463.

[22] H. J. Coles and C. Strazielle: Molecular Crystals and Liquid Crystals Vol. 55 (1979), p.237.

[23] H. J. Coles and C. Strazielle: Molecular Crystals and Liquid Crystals Vol. 49 (1979), p.259.

[24] S. Urban, J. Przedmojski, and J. Czub: Liquid Crystals Vol. 32 (2005), p.619.

[25] N. M. Abukhdeir and A. D. Rey: International Association of Science

and Technology for Development (IASTED) 594-015, ACTA Press, 2007.

[26] G. Barbero and Luiz Roberto Evangelista: An Elementary Course on the Con-

tinuum Theory for Nematic Liquid Crystals (World Scientific Publishing Company, 2000).

[27] S. Chandrasekhar: Liquid Crystals, $2^{\text {nd }}$ ed. (Cambridge University Press, Cambridge, 1992).

[28] S. Pankratz, P. M. Johnson, R. Hołyst, and C. C. Huang: Phys. Rev. E Vol. 60 (1999), p. R2456.

[29] S. Pankratz, P. M. Johnson, A. Paulson, and C. C. Huang: Physical Review E Vol. 61 (2000), p.6689. 